\title{
Research on Dynamic Characteristic on the Circular Saw Blade of the Plastic Doors and Windows Corner Cleaning Based on ANSYS
}

\author{
Li Guoping ${ }^{1, \text { a }}$, Huang Zhenyong ${ }^{1, \mathrm{~b}}$, Zhong Wenzhen ${ }^{1, \mathrm{c}}$ \\ ${ }^{1}$ School of Mechanical Engineering, University of Jinan, Shandong Jinan 250022 \\ ame_ligp@ujn.edu.cn, ${ }^{b}$ hzy1006_me@163.com, ${ }^{c}$ me_zhongwz@163.com
}

Keywords: Profiles; Finite Element Analysis; Circular Saw Blades; Modal Analysis; Corner Cleaning

\begin{abstract}
In the plastic profiles corner cleaning process, the axial vibration of the end mill caused the corner cleaning quality is poor. So, it used the SolidWorks to establish the three-dimensional entity model of the circular saw blades in this article, and then the natural frequencies and the corresponding vibration types of the circular saw blades have been obtained from model analysis of the circular saw blade of the plastic doors and windows corner cleaning machines by using ANSYS, and the analysis of transverse vibration stability of the low frequency range. It was concluded that the allowable speed range of the circular saw blade was 1000rpm-6459rpm and a larger diameter chuck was utilized to enhance the vibration stability of the circular saw blade. The research aims to provide reference data for rationalization of the circular saw blade parameters selection and enterprise actual plastic doors and windows corner cleaning. It has an important theoretical value to improve the quality of the plastic doors and windows corner cleaning.
\end{abstract}

\section{Introduction}

In production of the plastic doors and windows, the actual corner cleaning process is accompanied by the dangerous state of high-speed rotation. And there are some problems are often occurred in the corner cleaning process, such as the edge breakage, the collapse edge and the fracture, etc.

Generally speaking, the instability of the circular saw blade is the transverse vibration of the circular saw blade ${ }^{[1]}$. Zou Lu ${ }^{[2]}$ thinks that the circular saw blade should avoid the rotational speed in the vicinity of its critical speed to avoid dynamic instability during the corner cleaning process. Wang Yazhou $^{[3]}$ thinks that the main reasons for the transverse vibration of the circular saw blade is the cyclical shocks and alternating stress which caused by intermittent cutting between the tool and the work piece. Xu Dongzhen ${ }^{[4]}$ thinks that the amplitude of lateral vibration in cleaning is smaller than free rotation.

So, it is necessary to know the natural frequency properties of the circular saw blade to avoid the circular saw blade produce resonance instability under the high speed rotation of NC shaft. And the profile corner cleaning is shown in Fig. 1. And the main performance parameters of the plastic doors and windows profile as shown in Table 1. 
Table 1 Performance parameters of the profile

\begin{tabular}{|ll|ll|}
\hline \multicolumn{1}{|c|}{ Project } & \multicolumn{1}{c|}{ Target } & \multicolumn{1}{c|}{ Project } & Target \\
\hline Heat Transfer Coefficient, $\mathrm{W} / \mathrm{m} 2 \cdot \mathrm{K}$ & $\geq 1.91$ & Melting Point, ${ }^{\circ} \mathrm{C}$ & $\geq 210$ \\
Elastic Modulus, MPa & $\geq 3000$ & Poisson's Ratio & 0.40 \\
Bending Modulus of Elasticity, $\mathrm{MPa}$ & $\geq 100$ & Density, $\rho /\left(\mathrm{kg} \cdot \mathrm{m}^{3}\right)$ & 1385 \\
\hline
\end{tabular}

\section{Modal Analysis of the Circular Saw Blade}

Because of the power of lateral vibration is mainly concentrated in the low frequency range $(<2$ $\mathrm{kHz})^{[5]}$. Therefore, this article mainly analyzes the transverse vibration of the circular saw blades in the low frequency range.

\subsection{The Finite Element Analysis Model of the Vibration}

In the corner cleaning process, the circular saw blades and the coupling of the shaft is fixed constraint. The vibration of the circular saw blade model as shown in Fig.2, the shadow part of the figure for the circular saw blade chuck, $\mathrm{R}_{1}$ as the radius of the clamp, $\mathrm{R}_{2}$ as the radius of the circular saw blade; $\mathrm{H}$ is the thickness of the circular saw blade.

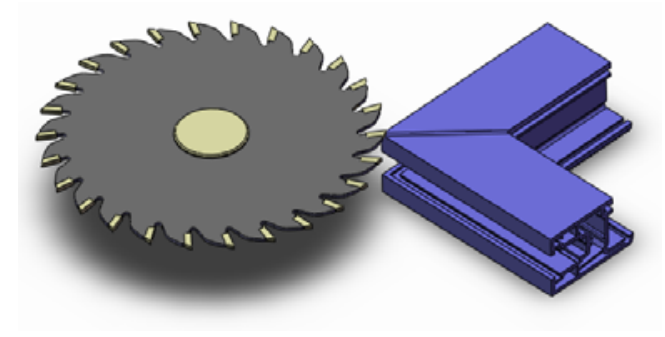

Fig.1 Profile corner cleaning

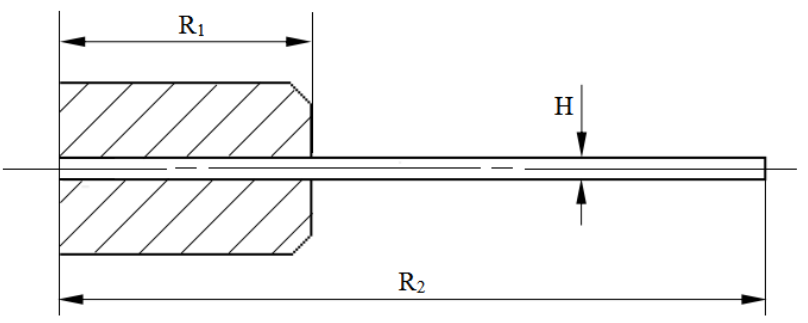

Fig.2 The circular saw blade vibration model diagram

According to the elastic theory, the nth-order natural frequency can be calculated by the formula:

$$
\omega=\frac{\alpha_{\mathrm{n}}}{2 R^{2}} \sqrt{\frac{E \mathrm{t}^{3}}{3\left(1-\mu^{2}\right) \rho}} .
$$

In the formula, $\omega$ is the nth-order modal constants; $t$ is the thickness of the circular saw blade; $\mathrm{R}$ is radius of the circular saw blade; $\mathrm{E}$ is the modulus of elasticity of circular saw blade material; $\mu$ is Poisson's ratio; $\rho$ is the material density.

It can be seen from the formula that there are many discrete natural frequencies of the circular saw blade, but only the low frequency vibration has great energy. And the natural frequencies of the circular saw blade will be increases with its thickness increasing. The properties of the material will be determined when the blade has been selected. So, in this section, the change of the natural frequency will be considered through change the geometric parameters of the circular saw blade.

\subsection{The Main Parameters of the Circular Saw Blade}

The circular saw blade used in this paper has a diameter of $200 \mathrm{~mm}$, the distance between the center hole and the tooth tip is $100 \mathrm{~mm}$, the diameter of the chuck is $50 \mathrm{~mm}$ and the thickness is $4 \mathrm{~mm}$. The dimensions and material parameters are shown in Table 2. 
Table 2 Material parameters of the circular saw blade

\begin{tabular}{|lcc|lcc|}
\hline Name & Unit & Numerical & Name & Unit & Numerical \\
\hline Diameter & $\mathrm{mm}$ & 200 & Density & $\mathrm{Kg} / \mathrm{m}^{3}$ & 7850 \\
Chuck Diameter & $\mathrm{mm}$ & 50 & Elastic Modulus & $\mathrm{GPa}$ & 206 \\
Thickness & $\mathrm{mm}$ & 4 & Material & & $65 \mathrm{Mn}$ \\
\hline
\end{tabular}

\subsection{The Analysis of the Circular Saw Blade's Natural Frequency \& Vibration mode}

There are many order particular vibration modes, its vibration mode and corresponding resonance frequency is fixed for a specific vibration modal. Resonance frequency is the natural frequency and the single vibration mode will appear when the circular saw blade vibration ${ }^{[6]}$.

\subsubsection{Circular Saw Blade Model Creation and Meshing}

It will be used the SolidWorks to establish the model of the circular saw blade, and import the model into ANSYS to mesh. The meshed model is shown in Fig.3.

\subsubsection{Loading Constraints and Solving}

Due to the existence of the chuck, it is necessary to apply the displacement constraint to all nodes in the central hole of the circular saw blade in radial, tangential and axial directions. The displacement of the center hole is constrained as shown in Fig.4.

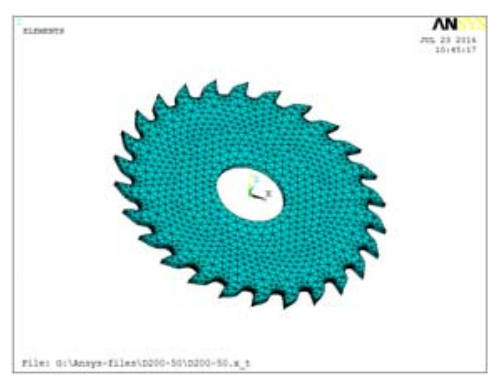

Fig.3 The meshed model

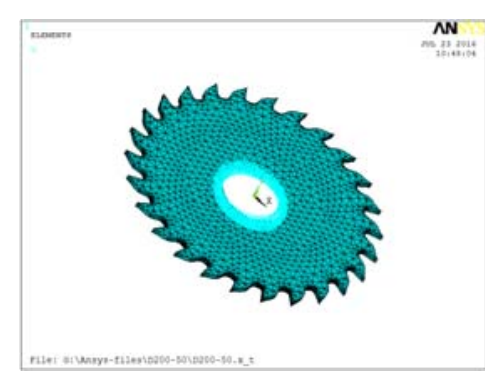

Fig.4 The displacement of the center hole is constrained

\subsubsection{Modal Analysis Results}

The natural frequencies of each order and the corresponding main vibration pattern are obtained through the modal analysis of the circular saw blade. Table.3 and Fig.5 are the natural frequencies values of the first ten orders of the circular saw blade and its main vibration mode of the circular saw blade.

Table 3 The natural frequencies values $(\mathrm{Hz})$

\begin{tabular}{|cc|cr|}
\hline Num & Frequency & Num & Frequency \\
\hline 1th-order & 725.11 & 6th-order & 1452.9 \\
2th-order & 725.11 & 7th-order & 1452.9 \\
3th-order & 743.64 & 8th-order & 2361.9 \\
4th-order & 874.30 & 9th-order & 2361.9 \\
5th-order & 874.30 & 10th-order & 3471.2 \\
\hline
\end{tabular}




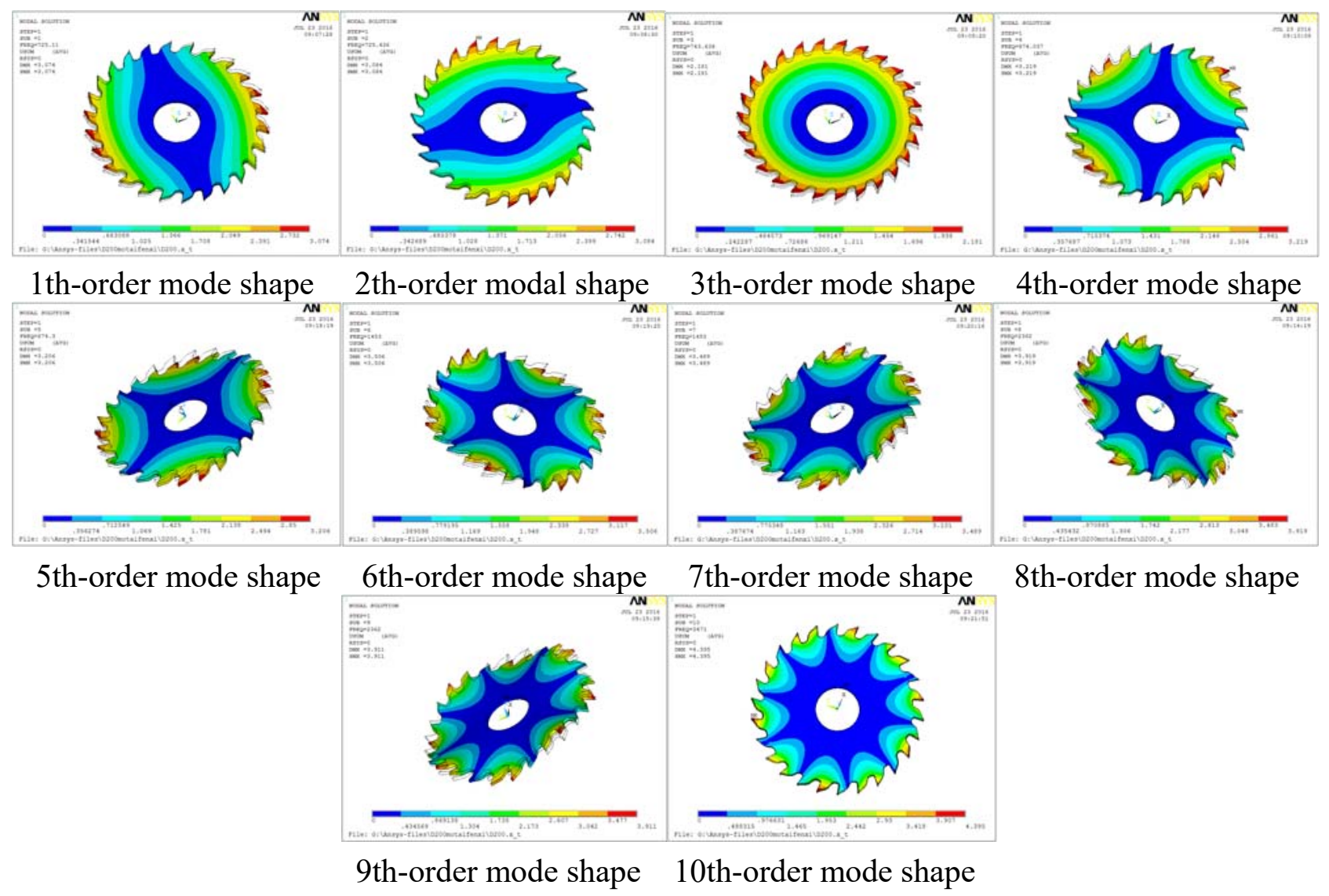

Fig. 5 The first ten orders mode shapes of the circular saw blade

From the Table 3 and Fig.5, it can be known that there is a rule which the central symmetry was shown in modal vibration figure, and it is conform to the structure characteristic of the saw blade itself. And the vibration mode of the First-order to the Third-order with a small amplitude oscillation, but the vibration mode will present the bending deformation obviously since the vibration mode of the Fourth-order, and the deformation of the vibration mode was increased due to the increase of the frequency.

According to the relationship between the circular saw blade critical speed and the frequency ${ }^{[7]}$ :

$$
\mathrm{n}=60 \omega / 2 \pi=60 \mathrm{f} \text {. }
$$

In this formula, $\mathrm{n}$ is the critical speed of the circular saw blade; $\omega$ is the angular velocity of the circular saw blade; $f$ is the natural frequency of the circular saw blade.

Because of the circular saw blade neglected modes corresponding to the rotational speed is higher. So, only the frequencies of the first four orders researched. The critical speed is shown in Table 4.

Table 4 The critical speed(rpm)

\begin{tabular}{|rcccc|}
\hline Num & 1th-order & 2th-order & 3th-order & 4th-order \\
\hline Speed & 43057 & 43057 & 44618 & 52458 \\
\hline
\end{tabular}

\subsubsection{Pre-stressed Modal Analysis}

Because of the high speed rotating circular saw blade will received the pre-stressed centrifugal force in the corner cleaning process. Therefore, the pre-stressed modal analysis was performed at the speed of $1000 \mathrm{rpm}, 2000 \mathrm{rpm}, 3000 \mathrm{rpm}, 4000 \mathrm{rpm}$. The natural frequencies of the first ten order under the action of centrifugal force as shown in Table 5. 
Table 5 The natural frequencies of the first ten orders under the action of centrifugal force $(\mathrm{Hz})$

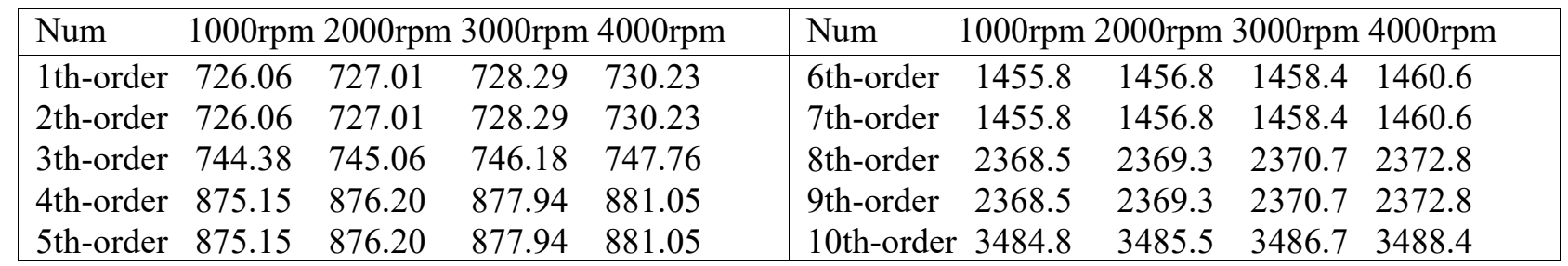

From the Table 5 , it can be seen that the change of the rotation centrifugal force has an influence on the natural frequency of the circular saw blade. And the higher rotational speed, the more obvious of the influence on the natural frequency of the circular saw blade. The critical speed of the first four orders of centrifugation is shown in Table 6.

Table 6 The critical speed of the first four orders of centrifugation(rpm)

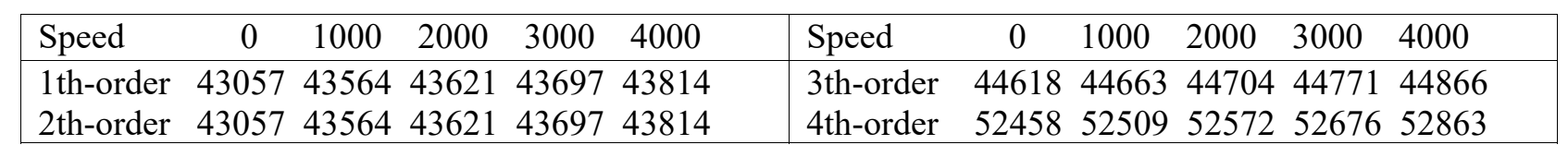

Generally speaking, the suitable working speed of the circular saw blade is lower than $15 \%$ of its critical speed. This value is named the minimum critical speed of the circular saw blade, and also the maximum rated speed ${ }^{[8]}$. Take the above four rotating speeds under the speed of the first order critical speed of $15 \%$ as the highest rated speed value of the circular saw blade to improve corner cleaning efficiency. The highest rated speed above four rotating speed as shown in Table 7.

Table 7 The highest rated speed above four rotating speed(rpm)

\begin{tabular}{|cccccc|}
\hline Speed & 0 & 1000 & 2000 & 3000 & 4000 \\
\hline 1th-order & 43057 & 43564 & 43621 & 43697 & 43814 \\
\hline
\end{tabular}

From the Table 7, it can be seen that the rotational speed of the circular saw blade itself is much lower than the maximum allowable rotational speed of the circular saw blade in the working state of each speed, taking $15 \%$ of the first critical speed of $43057 \mathrm{rpm}$ as the maximum speed of the circle saw blade. So, the speed value range for $1000 \mathrm{rpm}$ to $6459 \mathrm{rpm}$ of rotating circular saw blade should be choosed in the corner cleaning process. This range is not only less than the minimum of the critical speed value, but also to avoid the resonance phenomenon occurred. If the speed value of more than 6459rpm, need to combine the results of its modal analysis, to avoid the impact of clearance effect.

\subsection{The Effect of Clamp Diameter on the Natural Frequency of the Circular Saw Blade}

Yuan Ze, Cao Ruimin, Zhao Min ${ }^{[9]}$ pointed out that the natural frequency of the circular saw blade varies with its own geometric parameters or the change of center chuck in the modal analysis of diamond circular saw blade. In this section, the finite element modal analysis was performed at the chuck diameter of the circular saw blade of $50 \mathrm{~mm}, 70 \mathrm{~mm}$ and $90 \mathrm{~mm}$. The first ten orders natural frequencies of the three different diameter of the chuck were extracted as shown in Table 8.

Table 8 The first ten orders natural frequencies of the three different diameter of the chuck $(\mathrm{Hz})$

\begin{tabular}{|cccc|cccc|}
\hline Chuck Diameter & $50 \mathrm{~mm}$ & $70 \mathrm{~mm}$ & $90 \mathrm{~mm}$ & Chuck Diameter & $50 \mathrm{~mm}$ & $70 \mathrm{~mm}$ & $90 \mathrm{~mm}$ \\
\hline 1th-order & 725.11 & 1024.6 & 1517.0 & 6th-order & 1452.9 & 1634.8 & 2054.4 \\
2th-order & 725.11 & 1033.4 & 1543.8 & 7th-order & 1452.9 & 1634.8 & 2054.4 \\
3th-order & 743.64 & 1033.4 & 1543.8 & 8th-order & 2361.9 & 2445.1 & 2724.3 \\
4th-order & 874.30 & 1169.6 & 1683.5 & 9th-order & 2361.9 & 2445.1 & 2724.3 \\
5th-order & 874.30 & 1169.6 & 1683.5 & 10th-order & 3471.2 & 3505.2 & 3664.1 \\
\hline
\end{tabular}

According to the Table 8, the relationship between the first ten orders natural frequencies with the 
chuck diameter changes can be drawn as shown in Fig.6.

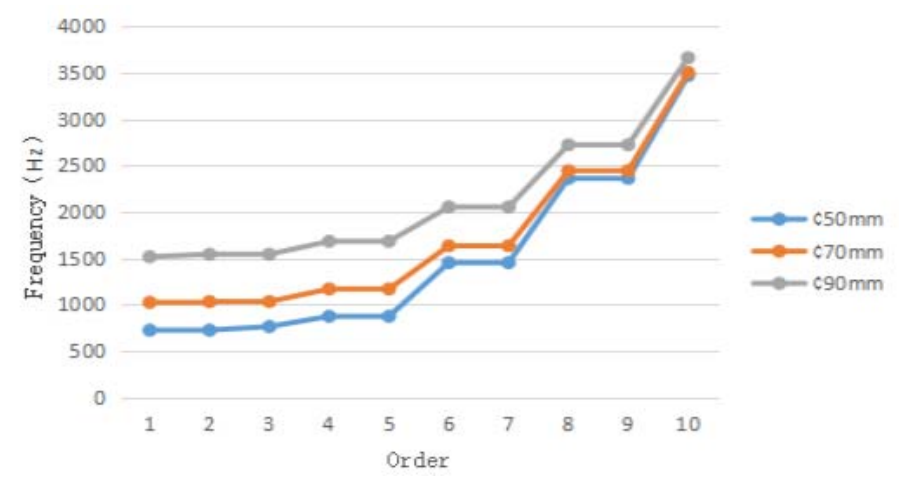

Fig.6 The relationship between the first ten orders natural frequencies with the chuck diameter changes

From the Fig.6, it can be seen that the natural frequencies of each order increase with the increase of the circular chuck diameter. And the first few orders natural frequencies change is significant. Therefore, the larger diameter chuck should be used to increase vibration stability of the circular saw blade in plastic doors and windows corner cleaning process.

\section{Conclusions}

The finite element modal analysis is carried out of the circular saw blade in plastic doors and windows corner cleaning process. And the following conclusions are:

(1) Through the finite element modal analysis method to calculate the natural frequencies and the main vibration mode of the circular saw blade. From the simulation results, it can be seen that we can choose the speed value range for 1000rpm to 6459rpm of rotating circular saw blade in the seam cleaning process to avoid the resonance phenomenon occurred. And it shows that the natural frequencies of the circular saw blade rotates at high speed is higher than the natural frequencies of the resting.

(2) The geometric parameters of the circular saw blade has a large effect on natural frequencies. And the influence of chuck diameter on vibration stability of the circular saw blade is analyzed. The natural frequencies of each order were increased with the increase of the circular chuck diameter. That is to say, the stiffness and stability will increase. So, the larger diameter chuck should be used to strengthen vibration stability of the circular saw blade in plastic doors and windows corner cleaning process.

In this article, the natural frequency and the corresponding vibration types of the circular saw blade has been obtained from model analysis of the circular saw blade of the plastic doors and windows corner cleaning machine by using the finite element analysis software (ANSYS), and the analysis of transverse vibration stability of the low frequency range. It was concluded that the allowable speed range of the circular saw blade was 1000rpm - 6459rpm, and a larger diameter chuck was utilized to enhance the vibration stability of the circular saw blade. The research aims to provide reference data for rationalization of circular saw blade parameters selection and enterprise actual plastic doors and windows corner cleaning. It has an important theoretical value to improve the quality of corner cleaning.

\section{Acknowledgements}

This paper was supported bythe Foundation for Outstanding Young Scientist in Shandong Province, Granted No. BS2015NJ009 


\section{References}

[1] Zhang Shaoqun, Jiao Guangze. Modal Analysis and Vibration Analysis of Circular Saw Blades Based on ANSYS. Forest Engineering, 2014, 30 (2):79-83.

[2] Zou Lu. Study on the Control of Transverse Vibration of Circular Saw Blade. Taiyuan: Taiyuan University of Technology, 2016.

[3] Wang Yazhou. Analysis and Research on the Vibration of the Saw Blade Cutter. Guangxi: Guangxi University of Science and Technology, 2015.

[4] Xu Dongzhen. The Study of Lateral Vibration of the Lamped Circular Saw in Cutting. Nanjing: Nanjing Forestry University, 2006.

[5] Wu Jiabin. Research on the Circular Saw's Dynamic Characteristic Basing the FEM. Tianjin: Hebei University of Technology, 2005.

[6] Li Xia. The Accuracy Analysis of Modal Synthesis Method. Xi'an: Xi'an Technological University, 2015.

[7] Jiang Lianqiong. Simulation Study of Circular Saw Cutting Process Based on ANSYS/LS-DYNA. Xi'an: Xi'an Technological University, 2015.

[8] Zang Yong, Li Tongjin, Tao Dengkui, etc. The Modal Analysis of Circular Saw Blade with FEM. Heavy Machinery, 2002 (1):49-52.

[9] Yuan Ze, Cao Ruiyuan, Zhao Min. Modal Analysis of Diamond Circular Saw Blade. Manufacturing Automation,2013 (9):147-149. 